\title{
Entrelacs
}

Cinéma et audiovisuel

18 | 2021

Laurent Roth, cinéaste

\section{Habiter le cinéma avec la grâce d'un poète en son jardin}

Julie Savelli

\section{OpenEdition}

Journals

Édition électronique

URL : https://journals.openedition.org/entrelacs/6068

DOI : $10.4000 /$ entrelacs.6068

ISSN : 2261-5482

Éditeur

Éditions Téraèdre

Référence électronique

Julie Savelli, « Habiter le cinéma avec la grâce d'un poète en son jardin », Entrelacs [En ligne], 18 | 2021, mis en ligne le 13 décembre 2021, consulté le 15 décembre 2021. URL : http://

journals.openedition.org/entrelacs/6068; DOI : https://doi.org/10.4000/entrelacs.6068

Ce document a été généré automatiquement le 15 décembre 2021.

Tous droits réservés 


\section{Habiter le cinéma avec la grâce d'un poète en son jardin}

\section{Julie Savelli}

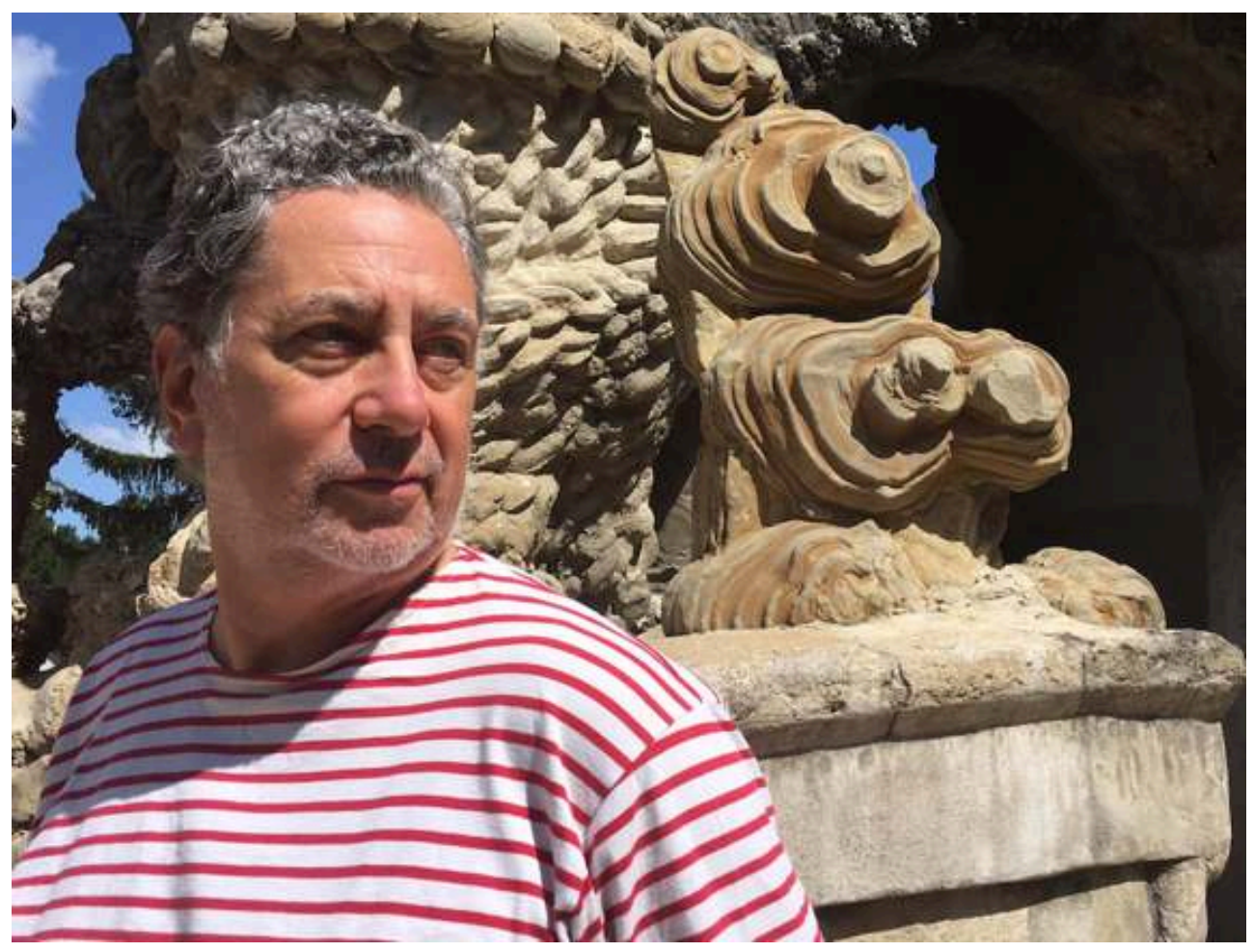

Laurent Roth à côté de la pierre d'achoppement (Palais du Facteur Cheval, Août 2021).

Crédit : Michèle Valentin.

Certains cinéastes, aussi importants soient-ils, demeurent confidentiels. Laurent Roth est de ceux-là : tout au long de son parcours, avec persévérance et continuité, avec modestie aussi, il a fabriqué ses films "à bas bruit », sans mettre en avant sa pratique cinématographique comme activité principale. Car Laurent Roth a de multiples cordes à son arc et il est assez rare d'occuper autant de fonctions différentes dans un parcours 
professionnel. Scénariste : il a collaboré à l'écriture des films de Jean-Daniel Pollet, Vincent Dieutre, Dominique Cabrera ou encore Stéphane Batut. Auteur dramatique : il a écrit une trilogie pour le théâtre - La Chose (2010), La Joie (2015), L'Âme (à venir) - et signé les livrets de l'opéra L'An Un et de l'oratorio Bar Iona de Jean-Christophe Marti. Critique de cinéma: il a notamment travaillé aux Cahiers du cinéma et pour France culture, publié une centaine d'articles et d'essais, ainsi que deux livres consacrés à Chris Marker et à Abbas Kiarostami ${ }^{1}$. Acteur : il apparait dans les films de ses complices, dont ceux de Jean-Julien Chervier, Abdelkrim Bahloul, Mathieu Amalric ou Peter Watkins. Enfin, Laurent Roth a également été pendant plusieurs années producteur au sein de la société Inthemood et directeur artistique de festivals (Festival International du documentaire «Fictions du réel » à Marseille, Ciné-citoyen à Paris). S'il n'a pas voulu faire profession de cinéaste - et n'a donc pas été véritablement célébré comme tel Laurent Roth a pourtant foi en le cinéma et cette foi est servie par de multiples talents. Occupé à défricher tranquillement son paysage intérieur, Laurent Roth habite le cinéma avec la grâce d'un poète en son jardin.

Quelles que soient les raisons de son invisibilité manifeste ${ }^{2}$ - démarche exploratoire, films à la première personne -, force est de constater que ce cinéaste appartient aux artisans des images, et non pas à l'industrie du film. Depuis Marie ou le retour - premier court métrage de fiction réalisé en 1984 avec de sublimes bouts de ficelles - jusqu'à L'Emmuré de Paris - fantaisie documentaire actuellement en post-production où le cinéaste incarne un autre-soi burlesque - en passant par le remontage d'archives de guerre, les essais tournés au Théâtre du Rond-Point avec Mireille Perrier et Mathieu Amalric, le remploi et la sonorisation de bobines achetées sur e-Bay ou encore la récente trilogie consacrée à Amos Gitaï ${ }^{3}$, les films de Laurent Roth sont des prototypes qui cherchent et trouvent un dispositif, avec des moyens minimalistes, pour réfléchir la relation entre l'image et la parole. Il en résulte autant de gestes et de partis pris qui confèrent à chaque production, aussi brève soit-elle, une fulgurance propre. En se renouvelant ainsi d'un projet à l'autre, les modes opératoires pointent la vitalité de l'acte de création tout autant que sa fragilité, laissant place à un cinéma qui expérimente les seuils. C'est donc avec la puissance d'un certain désœuvrement que le cinéaste Roth bricole à vue, s'attelant à assembler les pièces d'une œuvre patchwork aux couleurs de l'Arlequin, dont la singulière sensibilité touche autant qu'elle intrigue. 


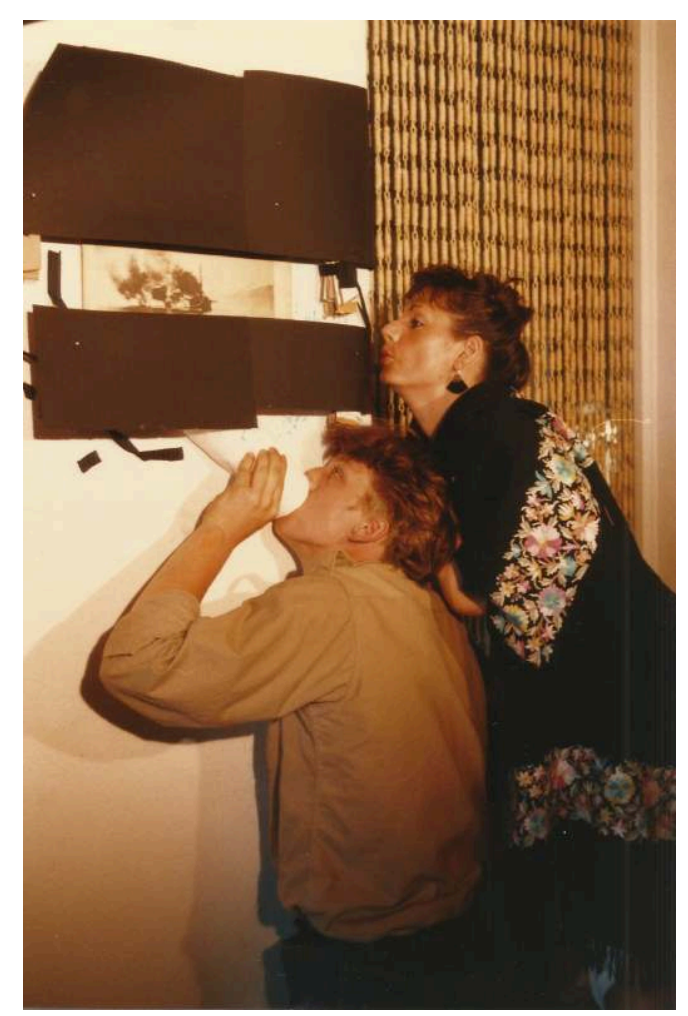

Marie ou le retour, 16mm, couleur et noir et blanc, 10', 1984. Fabrication de vraies-fausses archives de guerre (non montées) avec effet de fumée, par Emmanuel Maintigneux (ingénieur son) et Annette Coll (comédienne).

Crédit : Laurent Roth.

3 S'il est temps de saluer ce cheminement empreint de je(u), d'histoires (grandes et petites) et d'expérimentations (narratives, techniques, plastiques), il est nécessaire aussi d'éclairer, par un travail d'analyse et de documentation, la couture de cette grande toile à laquelle Laurent Roth travaille depuis près de quarante ans. En consacrant le présent numéro d'Entrelacs aux conduites créatrices de cet amator, nous aimerions contribuer à la reconnaissance d'une œuvre secrète qui offre la possibilité d'une autre voie, sans doute plus utopique, de faire et de penser en cinéma. Nourri par une variété d'expériences de réceptions, d'analyses et d'écritures, ce projeté éditorial ${ }^{4}$ offre une palette d'entrées dans l'œuvre du cinéaste, donnant la parole à des universitaires, à des artistes, amis ou collaborateurs. En parallèle, le sommaire présente également un ensemble de documents relatifs à la poiétique des films (entretiens, archives, correspondances, notes d'intention) ainsi que dix petits poèmes inédits constituant le recueil Poésie du seuil (1984), et trois critiques inaugurales de Laurent Roth - respectivement consacrées à Pierre Bonnard et à la figure de la porte-fenêtre, à sa pratique du film de famille en vidéo et à Persévérance ${ }^{5}$ de Serge Daney. Qu'elles relèvent d'une démarche littéraire, analytique ou génétique, les contributions ici réunies participent d'une scénographie de la disparité, laquelle s'est imposée d'ellemême en ce qu'elle découle des généalogies, tant matérielles qu'imaginaires, constituant la charpente de la maison-cinéma de Laurent Roth.

4 Nous tenons à remercier chaleureusement les auteur.e.s et les artistes qui ont contribué à la richesse de cet hommage - Antoine de Baecque, Dominique Cabrera, Roger-Philippe Della Noce-Bertozzi, Vincent Dieutre, Rémi Fontanel, Jacques Gerstenkorn, Yannick Haenel, Dario Marchiori, Lauren Roth, Michèle Valentin et Marc- 
Antoine Vaugeois - ainsi que les institutions scientifiques (Passages Arts \& Littératures XXe-XXIe à l'université Lyon 2, le RiRRa21 à l'université Montpellier 3, le LARA de l'université Toulouse Jean Jaurès) qui ont soutenu ce travail. Nous voulons enfin témoigner toute notre reconnaissance à l'écrivain Yannick Haenel, au peintre Michel Lascault et au cinéaste Laurent Roth dont la créativité, la Joie et la générosité ont conduit la mise en œuvre de ce projet en commun.

\section{NOTES}

1. Raymond Bellour et Laurent Roth, Qu'est-ce qu'une madeleine? À propos du CD-ROM Immemory de Chris Marker, Paris, Ed. Centre Georges Pompidou, «Les cahiers du musée national d'art moderne ", 1997 ; Alain Bergala, Jean-Michel Frodon, Laurent Roth et Charles Tesson, Abbas Kiarostami - Textes, entretiens, filmographie complète, Paris, Cahiers du cinéma, 2008.

2. Les films de Laurent Roth ne sont pas sortis en salles - à l'exception des Yeux brûlés (1986) distribué par Shellac après sa sélection au Festival de Cannes dans la section Cannes Classics en 2015 - mais ont été primés dans les grands festivals documentaires, programmés dans de nombreuses institutions culturelles (tels la BNF, le Centquatre, le Musée d'Art et d'Histoire du Judaïsme) ou encore présentés dans des manifestations universitaires.

3. Voir la filmographie de Laurent Roth dans le présent volume.

4. Ce projet éditorial a vu le jour à l'issue de la journée d'études "Le cinéma de Laurent Roth » organisée les 13 et 14 novembre 2019 par Jacques Gerstenkorn (Passages Arts \& Littératures XXeXXIe) à l'Université Lyon 2, et à laquelle Roger-Philippe Della Noce-Bertozzi, Rémi Fontanel, Dario Marchiori et Julie Savelli ont participé.

5. Serge Daney, Persévérance, Entretien avec Serge Toubiana, Paris, P.O.L., 1994.

\section{AUTEUR}

\section{JULIE SAVELLI}

Maîtresse de conférences en études cinématographiques et audiovisuelles à l'université PaulValéry Montpellier 3 et membre du Centre de recherches RiRRa21. Dans ses travaux qui portent sur la création documentaire contemporaine, elle s'intéresse plus particulièrement au cinéma engagé, à l'autobiographie en images et à l'anthropologie du visuel. Parmi ses diverses publications, mentionnons la direction de trois ouvrages collectifs en 2021 : Jean Rouch. Passeur d'images, passeur de mondes (Téraèdre), John Cassavetes. Imaginaires des corps, entre la scène et l'écran (PUP) et Dominique Cabrera. L'intime et le Politique (De l'Incidence Éditeur). 